REGARDS

SUR L'ECONOMIE ALLEMAND

BULLETIN ECONOMIQUE DU CIRAC
Regards sur l'économie allemande

Bulletin économique du CIRAC

$76 \mid 2006$

Varia

\title{
Mondial de football : panem et circenses
}

Isabelle Bourgeois

\section{OpenEdition}

Journals

Édition électronique

URL : http://journals.openedition.org/rea/822

DOI : $10.4000 /$ rea. 822

ISBN : 978-2-8218-0848-5

ISSN : 1965-0787

Éditeur

CIRAC

Édition imprimée

Date de publication : 1 mai 2006

Pagination : $37-38$

ISSN : 1156-8992

Référence électronique

Isabelle Bourgeois, « Mondial de football : panem et circenses », Regards sur l'économie allemande [En ligne], 76 | mai 2006, mis en ligne le 19 mai 2008, consulté le 15 septembre 2020. URL : http:// journals.openedition.org/rea/822 


\section{Actualité économique}

\section{- MONDIAL DE FOOTBALL : panem et circenses}

Le Coupe du Monde de la FIFA (9 juin-9 juillet) " n'aura rien d'un plan de relance » pour l'économie allemande. Contrant les espoirs des économistes qui en attendent un complément de croissance de 0,3\%, voire de 0,5\%, l'Institut der Deutschen Wirtschaft (IW), proche du BDI, se montre prudent. Une comparaison internationale des taux de croissance enregistrés les années de Coupe du Monde depuis ses débuts (1930) révèle en effet qu'il n'y a pas de relation de cause à effet identifiable entre ces événements et la croissance. Certes, le PIB français a crû de 3,6 \% en 1998, mais n'étaient-ce pas aussi les prémisses de l'effervescence liée à la bulle Internet ? En 1974, alors que l'Allemagne remportait le Mondial, elle n'enregistrait que $0,5 \%$ de croissance ; mais son économie subissait les effets du premier choc pétrolier...

L'enquête d'automne réalisée en 2005 par l'IW auprès des dirigeants d'entreprise nourrit elle aussi le scepticisme : la Coupe du Monde les laisse froids à $71 \%$. II est vrai qu'ils se préoccupent bien plus de leurs carnets de commande étrangers ; l'excédent commercial record enregistré en 2005 - 160,5 milliards $€$ - leur donne raison (les previsions 2006 sont tout aussi bonnes). Par ailleurs, le surplus attendu des activités touristiques liées au Mondial - maigre en comparaison : 1 milliard $€$ seulement - ira grossir la balance des paiements, non la balance commerciale... La consommation des ménages augmentera, bien sûr, de quelque 2 à 3 milliards $€$, mais c'est très peu, et ces dépenses profiteront exclusivement aux activités en rapport direct avec le ballon rond. Quant aux investissements liés à la modernisation des stades ou aux travaux d'infrastructures dans les hauts lieux du football (environ 6 milliards $€$, presque en totalité des fonds publics), ils ont déjà été effectués, comme le rappelle l'IW. Bref, les retombées économiques seront minces.

La Fédération des $\mathrm{CCl}$ allemandes, la DIHK, se montre, elle, plus optimiste ; elle s'attend à la création de 60000 emplois, soit autant que le ministère fédéral de l'Economie. Les branches concernées sont à l'évidence l'hôtellerie et la gastronomie, de même que la sécurité, qui devrait embaucher près de 15000 personnes. Le secteur de la mode outdoor et de l'équipement sportif n'est pas en reste; la société allemande Puma, par exemple, numéro 3 mondiale derrière Nike et Adidas, et qui produit les maillots de 12 équipes, met à profit l'événement pour une nouvelle stratégie marketing : il compte embaucher 300 salariés en Allemagne. Sur l'ensemble de tous ces emplois, un tiers devrait survivre au Mondial, " conséquence de l'amélioration des infrastructures et des perspectives de ventes à l'étranger liées aux effets positifs du Mondial sur l'image de l'Allemagne ", comme l'explique la DIHK. C'est peut-être là le principal bénéfice à attendre de la Coupe du Monde : un gain d'attractivité pour le Site Allemagne.

Cela étant, tout dépend des performances de l'équipe nationale sous la direction de son capitaine Jürgen Klinsmann, aussi controversé actuellement que l'était Aimé Jacquet en France avant que les Bleus ne gagnent. Les paris sont ouverts... Et ils dynamisent un secteur d'activité en marge de la légalité : celui des paris justement. Avec un CA de 1,5 milliards $€$ (le tiers seulement du CA britannique), il a un énorme potentiel de développement outre-Rhin. La fédération allemande des bookmakers s'attend à un doublement de ce CA à moyen terme - grâce notamment au Mondial de football. La banque publique du Land de Rhénanie-Palatinat table sur une volume total de 5 milliards $€$ en 2006. Curieusement, la récente affaire des matches truqués (" affaire Hoyzer ») avait certes consterné l'opinion, mais bien plus encore développé l'intérêt des Allemands pour les paris sportifs. L'institut Sport + Markt estime le nombre de parieurs à 7 millions actuellement, la clientèle potentielle à largement plus de 10 millions d'Allemands.

Mais le développement de ces activités est handicapé par un grand flou juridique. Théoriquement, le secteur des paris et des jeux de hasard est sous monopole d'Etat. L'unique opérateur dans le segment du football est la société ODDSET, filiale de la société publique de jeux de hasard Deutscher Lotto- und Totoblock (DTLB) ; elle figure parmi les sponsors du Mondial. Ce monopole s'étend à toutes les activités, sauf au pari hippique, un segment libéralisé en... 1922, et que se partage une centaine de bookmakers privés (recettes : 350 millions $€$ ). Or dans la pratique, le secteur des paris (tous sports confondus) s'assimile de facto à un marché, tant se sont multipliées les exceptions. Quatre bookmakers privés sont en concurrence: Betandwin, Goldesel/Digibet, Interwetten et Sportwetten Gera. Ces sociétés, dont les recettes sont estimées à quelque 500 millions $€$, avaient décroché une licence... en RDA, juste avant l'unification. Historiquement,
Le Mondial ne dopera pas la croissance allemande...

... mais accroîtra l'attractivité du site Allemagne

Il dynamisera le secteur des paris sportifs

Un grand flou juridique 
La Cour de Karlsruhe exige une réforme des paris sportifs

Le monopole ne peut pas se légitimer par des objectifs fiscaux

L'Office fédéral des Cartels s'en prend au monopole de la loterie leurs activités peuvent être jugées légales, bien qu'elles soient en contradiction avec l'état actuel du droit allemand. Parallèlement, en marge du droit national, la multiplication des paris sur Internet comme la diffusion outre-Rhin des activités des sociétés étrangères de loterie et de jeux de hasard contribue à l'essor d'un marché où œuvrent par ailleurs d'innombrables officines illégales.

Un sérieux toilettage du droit allemand s'impose d'urgence. De nombreux opérateurs potentiels sont dans les starting blocks, à commencer par l'opérateur de pay-TV Premiere qui a lancé à l'été 2005 une chaîne dédiée, à dominante hippique, qu'il projette d'étendre à l'ensemble des activités sportives et de diffuser 24 heures sur 24. La Fédération allemande du football DFB elle-même envisage de se diversifier dans les paris dès la saison 2006/07. Sans parler des géants scandinaves et britanniques des paris et de la lotterie qui attendent eux aussi la levée du flou juridique actuel.

Ils fondent leurs espoirs dans la jurisprudence. D'abord dans celle de la Cour européenne de justice, dont "l'arrêt Gambelli »(C-243/01) du 6-11-2003 avait considéré comme contraire à la liberté d'établissement et à la libre circulation des services au sein de l'UE la réglementation nationale restrictive (monopole) de l'Italie. Ensuite, sur celle du Tribunal constitutionnel fédéral allemand, saisi en dernier recours d'un contentieux opposant depuis près de dix ans une société désireuse de se diversifier dans les paris sportifs et l'Etat de Bavière. Dans son arrêt rendu le 28 mars 2006 (1 BvR 1054/01), il estime que le " monopole d'Etat des paris sportifs dans sa configuration actuelle n'est pas conciliable avec le droit fondamental de la liberté d'entreprise » et fait injonction aux législateurs de réformer la réglementation d'ici le 31-12-2007.

L'arrêt s'applique à tous les Länder. Seuls compétents en la matière, ils ont harmonisé leurs réglementations le 01-07-2004 en concluant un « Contrat d'Etat sur le secteur de la loterie en Allemagne », qui a dès lors force de loi sur l'ensemble du territoire. Ce texte justifie le monopole public par la protection des citoyens contre leur propension à l'autopréjudice : celui-ci vise en effet à " canaliser l'instinct de jeu naturel de la population dans des voies organisées et surveillées »(§1). Or les juges de Karlsruhe estiment cette justification quelque peu hypocrite : les activités de la société ODDSET en effet non seulement " poursuivent à l'évidence des objectifs fiscaux », mais concourent à entretenir l'image " d'une activité de loisirs foncièrement inoffensive ». Et ils rappellent que "les intérêts fiscaux de l'Etat ne constituent pas une justification pertinente pour un monopole public des paris sportifs ». Le législateur a donc deux possibilités : soit il maintient le monopole, mais alors il doit prendre des dispositions réelles et efficaces pour protéger les consommateurs, ce qui passe par l'adoption de critères définissant les activités liées aux paris et permettant de restreindre leur commercialisation; soit il libéralise le marché, mais en veillant à l'organiser grâce à l'attribution de licences d'exploitation.

Si les opérateurs privés existants et potentiels critiquent vertement cet arrêt qui annihile leurs perspectives commerciales dans le cadre du Mondial, les pouvoirs publics se frottent les mains, puisqu'il préserve le monopole d'ODDSET et leur garantit un surcroît de recettes fiscales. En années normale, le CA de cet opérateur s'élève à 400 voire 450 millions $€$ dont une partie alimente les budgets des Länder : 16,66 \% de taxe sur les jeux de hasard à quoi s'ajoute un impôt sur la concession dont le taux varie entre $15 \%$ et $20 \%$ selon le Land. La majeure partie de ces recettes est destinée au financement des activités sportives de masse. Les fédérations sportives plaident donc pour le maintien du monopole ou, à défaut, un régime de concessions - solution qu'adopteront vraisemblablement les législateurs, au nom de la promotion de la pratique sportive.

Leur réforme réglementaire s'appliquera à l'ensemble du secteur des jeux de hasard. Car si la Cour européenne de justice a ouvert la voie avec "l'arrêt Gambelli », mais aussi l'arrêt C-453/02 du 17 février 2005 rendu dans l'affaire opposant l'administration fiscale de la ville de Gladbeck et un exploitant de machines à sous, et si la Cour de Karlsruhe réclame la révision du droit des paris sportifs, l'Office fédéral des Cartels a à connaître depuis le début de l'année du monopole du Deutscher Lotto- und Totoblock. II avait été saisi par la société Jaxx (fliliale de la Fluxx AG) dont les 25500 bureaux du DTLB avaient refusé de diffuser les pronostics. Le commerce de détail (l'enseigne Edeka par exemple a lancé en avril 2005 une opération pilote en coopération avec la Fluxx $A G)$, tout comme les réseaux des stations services ou les opérateurs de TV, sont optimistes quant au verdict de l'Office des Cartels. Une ouverture du marché dopera la consommation. Mais une réforme de la législation n'interviendra qu'après le Mondial. Alors qu'en 2004, les recettes fiscales qu'ont tiré les pouvoirs publics des jeux de hasard se sont élevés à 4,2 milliards $€$, elles devraient enregistrer une nette hausse à l'occasion de l'événement - ce qui n'est pas sans déplaire aux ministres des Finances allemands. (IB) 